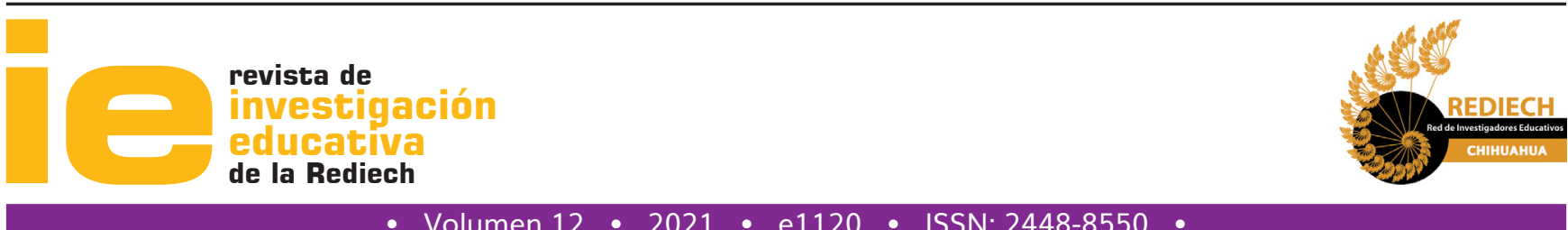

- Volumen 12 • 2021 • e1120 • ISSN: 2448-8550 •

\title{
Relación entre modelos educativos parentales y problemas de conducta en estudiantes
}

\author{
Relationship between parental educational models and behavioural problems in students
}

\author{
Laura Abellán Roselló
}

\begin{abstract}
RESUMEN
El objetivo de esta investigación consiste en indicar el modelo de crianza parental más utilizado y si este guarda relación con los problemas de conductas del alumnado, observados en el contexto educativo. La muestra estuvo compuesta por $206 \mathrm{pa}$ dres y/o madres de estudiantes con edades comprendidas entre los 4 y los 16 años $(\mathrm{M}=8,3$ y DT $=6,14)$, donde el $54,72 \%$ de los menores son hombres y el 45,28\% mujeres, cursando educación infantil $(17,92 \%)$, primaria $(54,72 \%)$ o secundaria $(29,24 \%)$. Los datos fueron recogidos mediante el Cuestionario de Crianza Parental y el ChildBehavior Checklist. Se llevó a cabo un estudio empírico-analítico, con carácter transversal, realizándose estudios descriptivos básicos y correlación lineal bivariada de Pearson. Los resultados mostraron que el modelo educativo parental más utilizado es el democrático. También se observa que el modelo autoritario se relaciona con problemas de conducta. Se comentan las implicaciones de estos hallazgos para la investigación y la práctica psicoeducativa.
\end{abstract}

Palabras clave: estilo educativo parental, prácticas de crianza, problemas conductuales, variables, adolescentes.

\begin{abstract}
The objective of this research is to indicate the most used parental upbringing model and if it is related to the students' behavior problems, observed in the educational context. The sample was composed of 206 fathers and/or mothers of students between the ages of 4 and $16(\mathrm{M}=8.3$ and $\mathrm{SD}=$ 6.14), where $54.72 \%$ of the children are men and $45.28 \%$ are women, studying in kinder school $(17.92 \%)$, elementary school $(54.72 \%)$ or middle school $(29.24 \%)$. The data was collected through the Parental Care Questionnaire and the ChildBehavior Checklist. An empirical-analytical study was carried out, with a transversal character, performing basic descriptive studies and bivariate linear correlation of Pearson. The results showed that the most widely used parental education model is the democratic one. It is also observed that the authoritarian model is related to behavioral problems. The implications of these findings for research and psychoeducational practice are discussed.
\end{abstract}

Keywords: parental education style, parenting practices, behavioral problems, factors, teenagers. 


\section{INTRODUCCIÓN}

La juventud de hoy en día está caracterizada por un significante rechazo hacia las normas y medidas de disciplina que establece la familia, probablemente como contraposición a los modelos autoritarios que muchos de los padres de ahora recibieron en su infancia. Esto está generando modelos de crianza caracterizados por una falta de autoridad y disciplina que, como consecuencia, se acompaña de un considerable aumento de los problemas de conducta en los niños (Urra, 2006). De hecho, detrás del fracaso escolar, el motivo más habitual de consulta es el comportamiento desobediente y rebelde del menor (Espada, Orgilés y Méndez 2004).

La familia juega un papel muy importante en el desarrollo de las personas, no solo porque garantiza su supervivencia física sino también porque es dentro de ella donde se realizan los primeros aprendizajes necesarios para desenvolverse autónomamente en la sociedad (Mabbe, Vansteenkiste, Brenning, De Pauw, Beyers y Soenens, 2019), a la vez que van moldeando las características psicológicas del individuo, como son el pensamiento, el lenguaje, afecto, adaptación y la formación de su autoconcepto (Beltrán y Pérez, 2000; Palacios, 1999b).

El desarrollo del alumnado está condicionado por factores como: forma de ser de la familia, ambiente, estructura familiar, entre otros (Nielsen, 2011). Las formas de actuar de los padres con sus hijos son individuales y varían según las diferentes culturas. Normalmente, se pueden analizar mediante dos variables: el afecto y el control (Palacios, 1999a), aunque pueden analizarse muchas más variables dentro de la forma de actuar de una familia (Gray y Steinberg, 1999; Slicker, Picklesimer, Guzak y Fuller, 1998; Kerr y Stattin, 2000; Stattin y Kerr, 2000; Iborra, 2017). Las combinaciones de esas variables dan como resultado los llamados "estilos parentales" (Davis, Thomassin, Bilms, Suveg, Shaffer y Beach, 2017).

El concepto "estilo parental" lo acuñó hace más de 30 años Baumrind (1973), estableciendo tres tipos de estilos educativos parentales utilizados por los padres para controlar el comportamiento de los hijos: el modelo autoritario, el permisivo y el democrático. Posteriormente Maccoby y Martin (1983) mediante sus investigaciones aportaron a la teoría de Baumrind (1973) un estilo más, ya que vieron que la permisividad se podía ver de dos maneras distintas, por lo que dividieron el estilo permisivo en dos: permisivo indulgente y permisivo negligente (indiferencia a los hijos y falta de supervisión), y establecieron una tipología de cuatro estilos parentales: autoritario,

Laura Abellán Roselló. Profesora-investigadora del Área Métodos de Investigación y Diagnóstico en Educación de la Universidad Internacional de la Rioja (España). Es doctora en Educación por la Universidad de Valencia (España). Sigue la línea de investigación de aprendizaje y motivación para mejorar la calidad educativa. Ha presentado sus contribuciones científicas en congresos nacionales e internacionales, así como a través de publicaciones científicas. Correo electrónico: laura.abellan@unir. net. ID: https://orcid.org/0000-0003-3009-9024. 
democrático, permisivo e indiferente. Esto se hizo con base en dos ejes: el grado de sensibilidad o afecto que muestran los padres hacia los hijos, y la exigencia y control que los padres ejercen sobre sus hijos. En relación a esto, algunos estudios (Ha, Otten, McGill y Dishion, 2019; Wetterborg, Enebrink, Lönn Rhodin, Forster, Risto, Dahlström y Ghaderi, 2019) han demostrado que el que mejores consecuencias trae para el estudiantado es el autoritario, consiguiendo en estos mayor madurez y competencia.

Con el tiempo, más autores sugirieron la importancia de más variables como la autonomía, sobre todo a la hora de analizar el estilo democrático (Van Heel, Van Den Noortgate, Bijttebier, Colpin, Goossens, Verschueren y Van Leeuwen, 2019; Slicker et al., 1998; Gray y Steinberg, 1999); la revelación (Kerr y Stattin, 2000; Stattin y Kerr, 2000; Wittig y Rodriguez, 2019), es decir, el acto espontáneo de los hijos de contar a los padres las actividades de la calle o sus amistades entre otros, es decir, lo que se hace cuando ellos no están; esta variable hace que tengan buen comportamiento y puede ser considerada como una estrategia sutil de control parental que prevenga algunos comportamientos de riesgo (Crouter, Bumpus, Davis y McHale, 2005; Parra y Oliva, 2006). Según Dunn (1993), otra variable puede ser el humor, puesto que describe la relación entre padres e hijos, ya que el humor puede crear un clima familiar favorable que haga más eficaces los intentos socializadores de los padres y promueva el bienestar de niños y adolescentes (Darling y Steinberg, 1993; Parkes, Green y Mitchell, 2019).

Por todo ello, los estilos de crianza parental son las formas características de interacción que tienen los padres hacia sus hijos (Woolfolk, 2010). El equilibrio en la interacción familiar, fundamental para la crianza, se consigue empleando afecto, comunicación y, por otra parte, una disciplina que incluya límites y normas de comportamiento claras (Darling y Steinberg, 1993; Maughan y Barker, 2019). Aunque según Nielsen (2011), habría una serie de factores que podrían modificar los patrones de comportamiento de los padres en la crianza.

La mayoría de los estudios encuentran que los estilos democráticos son más frecuentes cuando el nivel socioeconómico es alto (Baer, 1999; Roy, Isaia y Li-Grining, 2019), por lo que las prácticas parentales pueden representar un claro mediador de la relación entre este factor y el desarrollo infantil y adolescente (Bradley y Corwyn, 2002).

Según estudios basados en la relación entre la estructura familiar y el estilo parental, se encuentra una mayor frecuencia de estilos democráticos en familias tradicionales que en familias reconstituidas (Slicker et al., 1998) o monoparentales (Baer, 1999; Pettit, Laird, Dodge, Bates y Criss, 2001). Por otro lado, si los padres tienen conflictos maritales, debido al malestar que vive la pareja, generará prácticas de crianza perjudiciales, las cuales ocasionan un ambiente no muy bueno para el desarrollo de los hijos (Moreno, 2013; Wittig y Rodriguez, 2019).

Además, se ha podido comprobar que los hijos y las hijas reaccionan y perciben las estrategias educativas de sus padres de una manera diferente, sobre todo en la 
adolescencia, ya que en esta etapa son más evidentes los cambios que se producen en la percepción de sí mismos, de los padres y de las relaciones de ambos (Higgins, Ruble y Hartup, 1983). Algunos trabajos describen diferencias entre chicos y chicas, señalando por ejemplo que ellas se sienten más controladas que sus compañeros varones (Baumrind, 1991; Del Barrio y Carrasco, 2004; Dornbush, Ritter, Leiderman, Roberts y Fraleigh, 1987; Moreno, Muñoz, Pérez y Sánchez-Queija, 2004; Shek, 2000). Por otro lado, en unos estudios realizados por Fuentes, Motrico y Bersabé (2001) demostraron que los niños eran los que más rechazados y criticados se sentían en comparación con las niñas, sentían que sus padres eran más estrictos con ellos, les imponían el cumplimiento de unas normas y exigían demasiado. Sin embargo, en otros trabajos no encuentran diferencias de género (Smetana, 1995; Mabbe et al., 2019).

En cuanto a las relaciones de los adolescentes con sus madres y sus padres, se ha estudiado que son bastante diferentes (Collins y Russell, 1991). Las madres suelen mantener más conversaciones con sus hijos/as adolescentes (Noller y Callan, 1990; Fuentes, Motrico y Bersabé, 2001), estos las perciben más cercanas emocionalmente y por eso se convierten en una fuente de apoyo emocional esencial (Miller y Stubblefield, 1993; Khafi, Borelli y Yates, 2019).

Por último, la edad puede hacer que los padres utilicen un estilo u otro debido a que, a medida que la edad de los hijos va aumentando, los padres sienten que pierden el control y recurren a técnicas más controladoras, utilizando la imposición ante la reflexión y el diálogo que utilizan cuando las edades son más tempranas (Palacios, 1999a; Jung, Zhang, Hwang y Zhang, 2018). Por otro lado, la mayoría de los estudios demuestra que a lo largo de la adolescencia los padres y madres disminuyen los niveles de control, adaptando sus prácticas educativas a las necesidades e intereses de los adolescentes (Parra y Oliva, 2006; Collins y Steinberg, 2006; Parra, 2005; Moreno et al., 2004; Shek, 2000).

Teniendo en cuenta estas variables y tal y como se ha estudiado (Jung et al., 2018; Van Heel et al., 2019), el rechazo parental es un importante predictor en la aparición de muchos de los problemas del comportamiento infantil, como los trastornos de conducta, comportamiento negativista desafiante, delincuencia, esquizofrenia, trastornos de ansiedad, trastornos de la alimentación o abuso de drogas y alcohol (Pino, Menchón, Mataix-Cols, Pifarré, Urretavizcaya, Crespo, Vallejo y Vallejo, 2004; Bradford, Barber, Olsen, Maughan, Erickson, Ward y Stolz, 2004; Khaleque y Rohner, 2002, 2012; Rohner y Britner, 2002). Muchos estudios longitudinales han demostrado que el rechazo de los padres suele ser un precedente al desarrollo de los problemas psicopatológicos (Ge, Best, Conger y Simons, 1996; Ge, Lorenz, Conger, Elder y Simons, 1994; Loeber y Stouthamer-Loeber, 1986; Petersen, Sarigiani y Kennedy, 1991; Simons y Robertson, 1989), por lo que es probable que el rechazo que se percibe 
en los padres sea un mecanismo generativo responsable directa e indirectamente del desarrollo de diferentes alteraciones psicopatológicas.

Sin embargo, hoy en día, debido al estilo de vida que lleva la sociedad, las familias apenas tienen tiempo para dedicar a sus hijos, por lo que las formas de educar a los menores han cambiado (Rodríguez, 2010). Ahora los dejan en centros escolares esperando que se encarguen de su educación además de desarrollarles en los demás ámbitos (intelectual, físico, psicomotor y socio-afectivo).

Por todo esto, se ve la necesidad de buscar la relación que existe entre los problemas de conducta del alumnado y el estilo de crianza parental llevado a cabo con ellos. Estudios que relacionan el clima social familiar y conductas agresivas de los adolescentes señalan como factores de riesgo a los hogares con alto nivel de conflicto, con escasa relación afectiva entre sus miembros y bajo apoyo emocional (Lösel y Farrington, 2012). Según Bowlby (1985), la calidad de las relaciones del estudiante en su familia incide en sus relaciones tanto prosociales como de riesgo. Cuanto mayor sea la calidad de las relaciones familiares, menor es el impacto antisocial. Estudios que han contemplado aspectos como la falta de supervisión y compromiso con la crianza, la sitúan, junto con unas pautas disciplinarias inconsistentes, como un factor determinante de la conducta agresiva en los hijos (Knutson, DeGarmo y Reid, 2004; Jung et al., 2018; Mazefsky y Farell, 2005; McCoy, Frick, Loney y Ellis, 1999).

En conclusión, la mayoría de las investigaciones revisadas indican que existe gran influencia de los modelos educativos parentales en la conducta de sus hijos. Sin embargo, se puede observar en el día a día que no todos los hijos/as de padres autoritarios, o no todos los hijos/as de padres permisivos, cumplen las características que dichos estudios han señalado. Además, no se han encontrado trabajos donde se relacionen los modelos educativos parentales con variables tan importantes como edad o sexo, por lo que es importante seguir investigando para determinar cómo mejorar las relaciones parento-filiales.

\section{Metodología}

\section{Objetivo}

El objetivo de esta investigación consiste en indicar el modelo de crianza parental más utilizado y si este guarda relación con los problemas de conductas del alumnado, observados en el contexto educativo.

\section{Tipo de estudio}

Se llevó a cabo un estudio empírico-analítico, con carácter transversal. La investigación fue expost facto con un nivel de indagación relacional, y la metodología empleada fue cuasi-experimental para dar respuesta al objetivo planteado. Se utilizó el paquete 
estadístico Statistical Package for the Social Science, versión 22.00 (SPSS Inc., 2018), para realizar los análisis estadísticos.

\section{Participantes}

La muestra del estudio se seleccionó siguiendo un procedimiento de muestreo aleatorio simple. La muestra de este estudio consta de 206 padres y/o madres de estudiantes cursando educación infantil $(17.92 \%)$, primaria $(52.83 \%)$ o secundaria $(29.24 \%)$, quienes participaron voluntariamente. La edad del alumnado oscila entre 4 y 16 años $(\mathrm{M}=8,3$ y DT $=6,14)$, siendo $54,72 \%$ de niños frente a $45,28 \%$ de niñas.

\section{Procedimiento}

Previo a la administración de pruebas se obtuvo autorización por parte de la Consellería de Educación, Cultura y Deporte, además del consentimiento informado de todos los progenitores participantes en este proyecto. Los instrumentos se facilitaron a través de un enlace a un cuestionario en línea a los padres/madres que voluntariamente quisieron colaborar. La administración de la batería de preguntas se llevó a cabo en un plazo de 15 días. Comprendía una sesión individual de 20 minutos. Los resultados se incorporaban directamente a una base de datos a la cual solo el personal autorizado tenía acceso, siendo toda la información proporcionada por los progenitores completamente anónima

\section{Materiales-instrumentos}

Para llevar a cabo el estudio se han utilizado adaptaciones de dos cuestionarios: de la adaptación española del Cuestionario de Crianza Parental (PCRI; Gerard, 1994) el cual mide actitudes de los padres hacia los niños, y el ChildBehavior Checklist (CBCL; Achenbach y Edelbroch, 1978, 1983), en el que los padres puntúan la conducta de sus hijos de 4 a 16 años en los últimos seis meses.

El CBCL estructura los problemas conductuales que se presentan en los niños en dos categorías primarias de trastornos: las agrupaciones de conducta internalizada o intradirigida y la de conducta externalizada o extradirigida. La primera agrupación incluye las dificultades psicológicas que se dirigen hacia el interior, cuando la perturbación del niño está en relación con su medio interno y sus síntomas fundamentales se asocian con lo que se ha dado en denominar "comportamientos sobrecontrolados". El cuestionario se conforma de nueve trastornos que son: la agresividad, la depresión, obsesivo-compulsivo, delincuencia, ansiedad somática, problemas somáticos, delincuencia no-socializada, hiperactividad y retraimiento social.

Este cuestionario ha sido adaptado, dando lugar a 40 ítems puntuados por los padres que describen el comportamiento específico de sus hijos y los problemas 
emocionales. No se pusieron ítems de cada trastorno porque se pretendía que el cuestionario fuese en 40 preguntas, apto para cualquier edad y eficaz en su brevedad, por lo que no recoge ítems relacionados con los trastornos obsesivo-compulsivo y problemas somáticos. Al tener claro el objetivo de querer relacionar los problemas de conducta con los modelos educativos, se quiso reflejar en el cuestionario conductas habituales propias de problemas conductuales que generan interés de relacionarlas con el modelo educativo parental recibido. Además hay un ítem abierto para, en el caso de que existan problemas adicionales, que los describan. Las puntuaciones serán con base en cómo de cierto es cada ítem en los últimos seis meses. La puntuación de estos ítems se realiza en una escala de tipo Likert de 3 puntos, siendo $0=$ falso o raramente, 1 = en parte o algunas veces, y 2 = cierto o casi siempre. Si la media de cada trastorno es mayor de 1 es que se da mínimo en alguna ocasión; si es por debajo de 1 , no existe tal problema conductual.

El PCRI consta de 31 ítems con cuatro opciones de respuesta (en total desacuerdo, en desacuerdo, de acuerdo y muy de acuerdo) distribuidos en tres escalas: comunicación (9 ítems), disciplina (12 ítems) y autonomía (10 ítems). La puntuación de estos ítems se realiza en una escala de tipo Likert de 4 puntos, asociando 0 al "muy de acuerdo" hasta 4 a "en total desacuerdo". El ítem 20, que corresponde a la escala de "disciplina”, se invirtió para que midiera la misma relación que los otros. A través de estas escalas se deducen los estilos educativos de los padres ya que se asocia normalmente el exceso de disciplina y falta de autonomía en el niño con el estilo autoritario; la ausencia de normas y reglas de disciplina con el permisivo, y la buena comunicación, autonomía en el niño y buena disciplina con el estilo democrático.

\section{Pruebas estadísticas realizadas}

En primer lugar, se ha realizado un análisis estadístico descriptivo en el cual se ha medido la tendencia central, la media, la varianza y desviación estándar, con el objetivo de ver cuál es el modelo educativo parental más utilizado en la muestra que se ha recogido.

En segundo lugar, se ha realizado un análisis de correlación lineal bivariado de Pearson para ver si existe o no una relación lineal entre variables que determinan el modelo educativo parental y los problemas de conducta más observados en el centro educativo.

\section{Resultados}

\section{Análisis descriptivo}

A la hora de analizar los resultados se procederá a observar en primer lugar cuál es el modelo educativo parental más utilizado por los padres/madres de la muestra recogida. 
Para ello se analizará la media de la variable "comunicación”, la de "disciplina” y la de “autonomía”. De esta manera se relacionará la buena comunicación, bajos niveles de castigos físicos y la concesión de autonomía, con progenitores democráticos (Becker, Peterson, Luria, Shoemaker y Hellmer, 1962). Por el contrario, si la comunicación es escasa y conceden a los hijos poca autonomía, será un modelo educativo autoritario. En el caso de ver baja comunicación, poca disciplina y concesión de total autonomía del niño, hablaremos de un estilo permisivo (ver tabla 1).

Tabla 1. Análisis descriptivo comunicación, disciplina y autonomía existente entre padres/madres-hijos/as.

\begin{tabular}{ccccccc} 
& $\mathrm{N}$ & Mínimo & Máximo & Media & Desviación estándar & Varianza \\
\hline Comunicación & 206 & 1,11 & 2,89 & 1,8377 &, 30742 &, 095 \\
\hline Disciplina & 206 & 1,58 & 3,83 & 2,7233 &, 37911 &, 144 \\
\hline Autonomía & 206 & 1,90 & 3,60 & 2,8057 &, 33518 &, 112 \\
\hline
\end{tabular}

Se observa que la media de la muestra obtenida en comunicación tiene una baja puntuación (1.84), por lo que, como se ha explicado anteriormente, existe una buena relación progenitores-hijos/as. En disciplina la puntuación es alta, por encima de 2 (2.72), por lo que existe disciplina en las relaciones, pero tampoco se observa excesivo control ya que no llega al 3. Por último, en autonomía la media obtenida es alta, por encima de 2 (2.81), pero, al igual que ocurre con la disciplina, no es excesivamente alta, por lo que son padres que dan autonomía a sus hijos, pero sin perder del todo el control. Por todo ello, el estilo democrático es el que más se da en la muestra de progenitores analizada.

\section{Correlación bivariada de Pearson}

Una vez que se ha obtenido el modelo educativo parental más utilizado en la muestra, se procede a analizar distintas variables que, según las hipótesis anteriormente propuestas, interfieren en el modelo educativo parental.

Para comprobar este objetivo se relacionará las variables del modelo educativo (comunicación, agresividad y disciplina) con distintos problemas de conducta sacados del formulario realizado para la investigación: agresividad, depresión, hiperactividad, ansiedad y retraimiento. En general, se obtuvieron correlaciones positivas y significativas entre las variables del modelo educativo y los problemas de conducta. Las correlaciones que más destacan son las variables de problemas de conducta autonomía, ansiedad e hiperactividad con la variable de modelo educativo comunicación; la variable de problema de conducta delincuencia con la variable de modelo educativo agresividad, y las variables de problemas de conducta autonomía, ansiedad y retraimiento social con disciplina (ver tabla 2). 
Tabla 2. Correlación bivariada de Pearson entre variables del modelo educativo y problemas de conducta.

\begin{tabular}{|c|c|c|c|c|c|c|c|c|c|}
\hline & 1 & 2 & 3 & 4 & 5 & 6 & 7 & 8 & 9 \\
\hline 1. Comunicación & 1 & & & & & & & & \\
\hline 2. Agresividad & ,133 & 1 & & & & & & & \\
\hline 3. Disciplina &, $588^{* *}$ &,- 053 & 1 & & & & & & \\
\hline 4. Autonomía &, $570^{* *}$ & 011 &, $581^{* *}$ & 1 & & & & & \\
\hline 5. Ansiedad &,$- 376^{* *}$ & ,135* &, $662^{* *}$ & $186^{*}$ & 1 & & & & \\
\hline 6. Hiperactividad &, $234^{* *}$ & $221^{*}$ &,- 073 & $267^{*}$ &, $598^{* *}$ & 1 & & & \\
\hline 7. Depresión &,- 049 &,- 071 &,$- 181^{*}$ & $178^{*}$ &, $358^{* *}$ &,- 063 & 1 & & \\
\hline 8. Delincuencia &,$- 178^{*}$ & $453^{* *}$ &,$- 232^{*}$ & $178^{*}$ &, $217^{* *}$ &, $376^{* *}$ &, $303^{* *}$ & 1 & \\
\hline 9. Retraimiento social &,- 002 &,- 040 &, $527^{* *}$ &, $381^{* *}$ &, $460^{* *}$ &, $250^{* *}$ &, $646^{* *}$ &, $320^{* *}$ & 1 \\
\hline
\end{tabular}

\section{Discusión}

Partiendo del objetivo general de este trabajo de investigación, el cual era comprobar el modelo de crianza parental más utilizado y si este guarda relación con los problemas de conductas del alumnado, observados en el contexto educativo, tras los análisis realizados se pueden exponer los siguientes hallazgos.

En la muestra analizada, los padres no rechazan a sus hijos, son padres la mayoría democráticos, tal y como se ha obtenido al hallar la media de los estilos educativos utilizados en esos participantes. Este resultado va en la línea de los estudios de Ali, Khaleque y Rohner (2015); Khaleque (2015), y Khaleque y Rohner (2012b), que afirman que las conductas no apropiadas que realizan los niños y las niñas son debidas a algún problema con el estilo de crianza que han desarrollado sus progenitores. Por lo tanto, el modelo educativo que sale de esta recogida de datos es un modelo en el que hay buena comunicación, existen normas, pero sin ser exigentes y estrictos, y se les concede a los hijos autonomía, pero sin que exista esa falta de supervisión ni de límites. Con todo esto, se concluye que los padres y madres muestrales utilizan la mayoría un modelo democrático, en consecuencia, según Gómez-Ortiz, Del Rey, Romera y Ortega-Ruiz (2015), el ajuste del adolescente será mejor.

Además se han encontrado fuertes correlaciones significativas entre el modelo educativo parental y los problemas de conducta medidos, sobre todo entre el modelo educativo autoritario y los problemas de conducta como ansiedad y delincuencia. Estudios como el de Parra y Oliva (2006) o el de Collins y Steinberg (2006) van en la misma línea, concluyendo que el rechazo continuo de los padres suele originar diversas alteraciones interiorizadas y/o exteriorizadas en sus hijos, como depresión, conducta suicida, ansiedad, agresividad, hostilidad y delincuencia. 
Con este estudio se ve la importancia de la familia, y en especial de los estilos de crianza, para comprender el desarrollo de conductas agresivas en los hijos/as. Como bien muestran diferentes estudios, el comportamiento de la familia con el niño/a influye en su autoestima y en su forma de ver la vida, por lo que genera un comportamiento en torno a la visión de la realidad producida en su entorno más cercano, la familia. Para prevenir las manifestaciones de comportamientos agresivos es necesario intervenir con las familias desde los centros educativos, a las cuales habrá que orientar de forma adecuada para poder identificar las prácticas de crianza que suelen utilizar y que mantienen los problemas de conducta de sus hijos, con el objetivo de iniciar un proceso de modificación de esta (Trenas, Osuna, Olivares y Cabrera, 2013).

Entre las ventajas de este estudio se encuentra la evaluación parental de varios ciclos educativos. No obstante, debe tenerse en cuenta una serie de limitaciones. En futuros trabajos se deberían incorporar más variables familiares y educativas, como nivel socioeconómico (Vite y Pérez, 2014), estructura familiar (Quezada, 2015; Medina, 2016; Vásquez, 2015) o género (Del Barrio y Roa, 2006; Moreno, Estévez, Murgui y Musitu, 2009; Shek, 2000; Fuentes, Motrico y Bersabé, 2001), que han demostrado su influencia en la relación estudiada. También deberían tenerse en cuenta factores como la motivación y el estilo atribucional de cada individuo y factores socioemocionales que han demostrado tener relación en el rendimiento académico y disminución de problemas de conducta en el entorno educativo.

El presente trabajo sugiere que el estilo de educación parental posee un papel relevante en el comportamiento dentro del centro de estudios tanto en etapas iniciales como en toda la educación obligatoria, por lo que podría concluirse que el currículo debería fundamentarse en la investigación rigurosa y de calidad, centrándose también en una comunicación de calidad entre la familia y el centro educativo que fomenten una evolución positiva en el rendimiento académico y el clima de la clase.

\section{REFERENCIAS}

Achenbach, T. (1978). Psychopathology of childhood: Research problems and issues. Journal of Consulting and Clinical Psychology, 46, 759-776.

Achenbach, T., y Edelbrock, C. (1983). Manual for the child behavior checklist and revised child behavior profile. Burlington: University of Vermont.

Ali, S., Khaleque, A., y Rohner, R. P. (2015). Influence of perceived teacher acceptance and parental acceptance on youth's psychological adjustment and school conduct: A cross-cultural meta-analysis. Cross-Cultural Research, 49(2), 204-224. doi: 10.1177/1069397114552769.
Baer, J. (1999). The effects of family structure and SES on family processes in early adolescence. Journal of Adolescence, 22, 341-354.

Baumrind, D. (1973). The development of instrumental competence through socialization. En A. D. Pick (ed.), Minnesota Symposium on Child Psychology (vol. 7). Minneapolis: University of Minnesota Press.

Baumrind, D. (1991). Parenting styles and adolescent development. En R. M. Lerner, A. C. Petersen y J. Brooks-Gunn (eds.), Encyclopaedia of adolescence (pp. 746-758). Nueva York: Garland Publishing, Inc. 
Becker, W. C., Petersen, D. R., Luria, Z., Shoemaker, D. J., y Hellmer, L. A. (1962). Relations of factors derive from parent-interview ratings to behavior problems of five years olds. Child Development, 33, 509-535.

Beltrán, J., y Pérez, L. (2000). Educar para el siglo XX. Madrid: CCS.

Bowlby, J. (1985). La separación afectiva. Paidós.

Bradford, K., Barber, B. K., Olsen, J. A., Maughan, S. L., Erickson, L. D., Ward, D., y Stolz, H. E. (2004). A multi-national study of interparental conflict, parenting, and adolescent functioning: South Africa, Bangladesh, China, India, Bosnia, Germany, Palestine, Colombia, and the United States. Marriage \& Family Review, 35(34), 107-137. doi: 10.1300/J002v35n03_07.

Bradley, R. H., y Corwyn, R. F. (2002). Socioeconomic status and child development. Annual Review of Psychology, 53, 371-399.

Collins, W., y Russell, G. (1991). Mother-child and father-child relationships in middle childhood and adolescence: a developmental analysis. Developmental Review, 11, 99-136.

Collins, W. A., y Steinberg, L. (2006). Adolescent development in interpersonal context. En W. Damon y N. Eisenberg (eds.), Handbook of child psychology. Vol. 3: Social, emotional, and personality development (3a. ed., pp. 1003-1067). Nueva York: Wiley.

Crouter, A. C., Bumpus, M. F., Davis, K. D., y Mchale, S. M. (2005). How do parents learn about adolescents' experiences? Implications for parental knowledge and adolescent risky behavior. Child Development, 76, 869-882.

Darling, N., y Steinberg, L. (1993). Parenting style as context: An integrative model. Psychological Bulletin, 113(3), 487-496. Obtenido de http://www.oberlin. edu/faculty/ndarling/lab/psychbull.pdf.

Davis, M., Thomassin, K., Bilms, J., Suveg, C., Shaffer, A., y Beach, S. R. H. (2017). Preschoolers' genetic, physiological, and behavioral sensitivity factors moderate links between parenting stress and child internalizing, externalizing, and sleep problems. Developmental Psychobiology, 59, 473-485.

Del Barrio, M. V., y Carrasco, M. A. (2004). Confluenciay discrepancia percibida por los hijos en los hábitos de crianza paternos y maternos. Trabajo presentado en el II Congreso Hispano-Portugués de Psicología. Lisboa, Portugal.
Del Barrio, M. V., y Roa, M. L. (2006). Factores de riesgo y de protección en agresión infantil. Acción Psicológica, 2(4), 39-65.

Dornbush, S. M., Ritter, P. L., Leiderman, P. H., Roberts, D. F., y Fraleigh, M. J. (1987). The relation of parenting style to adolescent school performance. Child Development, 58, 1244-1257.

Dunn, J. (1993). Young children close relationships: Beyond attachment. Newbury Park: Sage.

Espada, J. P., Orgilés, M., y Méndez, F. X. (2004). Detección temprana en salud infanto-juvenil. En A. González-Menéndez, J. R. Fernández-Hermida y R. Secades-Villa (coords.), Guía para la detección e intervención temprana con menores en riesgo (pp. 179-217). Oviedo: Colegio Oficial de Psicólogos.

Fuentes, M. J., Motrico, E., y Bersabé, R. M. (2001). Diferencias entre padres y adolescentes en la percepción del estilo educativo parental: afecto y normasexigencias. Apuntes de Psicología, 19(2), 235-250.

Ge, X., Best, K. M., Conger, R. D., y Simons, R. L. (1996). Parenting behaviors and the occurrence and co-occurrence of adolescent depressive symptoms and conduct problems. Developmental Psychology, 32, 717-731. doi: 10.1037/0012-1649. 32.4.717.

Ge, X., Lorenz, F. O., Conger, R. D., Elder, G. H., y Simons, R. L. (1994). Trajectories of stressful life events and depressive symptoms during adolescence. Developmental psychology, 30(4), 467-483. doi: 10.1037/00121649.30.4.467.

Gerard, A. (1994). Parent-child relationship inventory: Manual. Los Angeles: Westem Psychological Services.

Gómez-Ortiz, O., Del Rey, R., Romera, E.-M., y OrtegaRuiz, R. (2015). Los estilos educativos paternos y maternos en la adolescencia y su relación con la resiliencia, el apego y la implicación en acoso escolar. Anales de Psicologia, 31(3), 979-989.

Gray, M. R., y Steinberg, L. (1999). Unpacking authoritative parenting: Reassessing a multidimensional construct. Journal of Marriage \& The Family, 61, 574-588.

Ha, T., Otten, R., McGill, S., y Dishion, T. J. (2019). The family and peer origins of coercion within adult romantic relationships: A longitudinal multimethod study across relationships contexts. Developmental Psychology, 55(1), 207-215. https://doi.org/10.1037/ dev0000630. 
Higgins, E., Ruble, D., y Hartup, W. (1983). Social cognition and social development. Cambridge: Cambridge University Press.

IBM (2018). IBM SPSS Statistics for Windows, Version 22,0. Armonk, NY: IBM Corp.

Iborra, I. (2017). El modelo de los tres ejes: modelos educativos familiares. Valencia: Universidad Católica de Valencia. Recuperado de: https://online.ucv.es/resolucion/ el-modelo-de-los-tres-ejes-modelos-educativosfamiliares-por-isabel-iborra/.

Jung, E., Zhang, Y., Hwang, W., y Zhang, Y. (2018). Parental health and children's functioning in immigrant families: Family roles and perceived treatment at school. Journal of Child \& Family Studies, 27(6), 18991913. https://doi.org/10.1007/s10826-018-1032-5.

Kerr, M., y Stattin, H. (2000). What parents know, how they know it, and several forms of adolescent adjustment: Further support for a reinterpretation of monitoring. Developmental Psychology, 36, 366-380.

Khaleque, A. (2015). Perceived parental neglect, and children's psychological maladjustment, and negative personality dispositions: A meta-analysis of multicultural studies. Journal of Child and Family Studies, 24, 1419-1428. doi: 10.1007/s10826-014-9948-x.

Khaleque, A., y Rohner, R. P. (2002). Reliability of measures assessing the pancultural association between perceived parental acceptance-rejection and psychological adjustment: A meta-analysis of cross-cultural and intracultural studies. Journal of Cross-Cultural Psychology, 33, 127-140. doi: 10.1177/0022022102033001006.

Khaleque, A., y Rohner, R. P. (2012). Pancultural associations between perceived parental acceptance and psychological adjustment of children and adults: A meta-analytic review of worldwide research. Journal of Cross-Cultural Psychology, 43, 784-800. doi:10.1177/0022022111406120.

Khafi, T. Y., Borelli, J. L., y Yates, T. M. (2019). Prospective associations between maternal self-sacrifice/ overprotection and child adjustment: Mediation by insensitive parenting. Journal of Child and Family Studies, 28(1), 202-217. https://doi.org/10.1007/ s10826-018-1245.

Knutson, J. F., DeGarmo, D. S., y Reid, J. B. (2004). Social disadvantage and neglectful parenting as precursors to the development of antisocial and aggressive child behavior: Testing a theoretical model. Aggressive Behavior, 30, 187-205.

Loeber, R., \& Stouthamer-Loeber, M. (1986). Family factors as correlates and predictors of juvenile conduct problems and delinquency. En M. Tonry y N. Morris (eds.), Crime and justice (pp. 29-149). Chicago, IL: University of Chicago Press.

Lösel, F., y Farrington, D. P. (2012). Direct protective and buffering protective factors in the development of youth violence. American Journal of Preventive Medicine, 43, S8-S23. Recuperado de https://www.ajpmonline. org/article/S0749-3797(12)00338-8/fulltext.

Maccoby, E. E., y Martin, J. A. (1983). Socialization in the context of the family: parent-child interaction. En E. M. Hetherington y P. H. Mussen (eds.), Handbook of child psychology. Vol. IV: Socialization, personality and social development (4a. ed., pp. 1-101). Nueva York: Wiley.

Mabbe, E., Vansteenkiste, M., Brenning, K., De Pauw, S., Beyers, W., y Soenens, B. (2019). The moderating role of adolescent personality in associations between psychologically controlling parenting and problem behaviors: A longitudinal examination at the level of within-person change. Developmental Psychology, 55(12), 2665-2677. https://doi.org/10.1037/dev0000802.supp.

Maughan, B., y Barker, E. D. (2019). The earlier the better? Pausing for thought.... Child Development, 90(1), 20-24. https://doi.org/10.1111/cdev.13168.

Mazefsky, C. A., y Farell, A. D. (2005). The role of witnessing violence, peer provocation, family support, and parenting practices in the aggressive behavior of rural adolescents. Journal of Child and Family Studies, 14(1), 71-85.

McCoy, M. G., Frick, P. J., Loney, B. R., y Ellis, M. L. (1999). The potential mediating role of parenting practices in the development of conduct problems in a clinicreferred sample. Journal of Child and Family Studies, 8(4), 477-494.

Medina, R. (2016). Estilos de crianza parental en niños de familias con violencia intrafamiliar [Tesis de Maestría]. Cuenca, Ecuador: Universidad de Cuenca. Recuperado de: http://dspace.ucuenca.edu.ec/handle/123456789/24046.

Miller, J. B., y Stubblefield, A. (1993). Parental disclosure from the perspective of late adolescents. Journal of Adolescence, 16, 439-455. 
Moreno, M. C., Muñoz, M. V., Pérez, P., y Sánchez-Queija, I. (2004). Los adolescentes españoles y su salud. Madrid: Ministerio de Sanidad y Consumo.

Moreno, D., Estévez, E., Murgui, S., y Musitu, G. (2009). Relación entre el clima familiar y el clima escolar: El rol de la empatía, la actitud hacia la autoridad y la conducta violenta en la adolescencia. International Journal of Psychology and Psychological Therapy, 9(1),123-136.

Moreno, N. (2013). Familias cambiantes, paternidad en crisis. Psicología desde el Caribe, 30(1), 177-209. Obtenido de http:/ /www.redalyc.org/articulo.oa?id=21328600009.

Nielsen, L. (2011). Shared parenting after divorce: A review of shared residential parenting research. Journal of Divorce \& Remarriage, 52(8), 586-609.doi: 10.1080/10502556.2011.619913.

Noller, P., y Callan, V. (1991). The adolescent in the family. Londres: Routledge.

Palacios, J. (1999a). La familia y su papel en el desarrollo afectivo y social. En I. Etxebarría, M. J. Fuentes, F. López y M. J. Ortiz (coords.), Desarrollo afectivo y social (pp. 267-284). Madrid: Pirámide.

Palacios, J. (1999b): La familia como contexto de desarrollo humano. Sevilla: Universidad de Sevilla.

Parkes, A., Green, M., y Mitchell, K. (2019). Coparenting and parenting pathways from the couple relationship to children's behavior problems. Journal of Family Psychology, 33(2), 215-225. https://doi.org/10.1037/ fam0000492.

Parra, A. (2005). Familiay desarrollo adolescente: un estudio longitudinal sobre trayectorias evolutivas [Tesis de Doctorado no publicada]Sevilla: Universidad de Sevilla.

Parra, A., y Oliva, A. (2006). Un análisis longitudinal sobre las dimensiones relevantes del estilo parental durante la adolescencia. Infancia y Aprendizaje, 29, 453-470.

Petersen, A. C., Sarigiani, P. A., y Kennedy, R. E. (1991). Adolescent depression: Why more girls? Journal of Youth and Adolescence, 20, 247-271. doi: 10.1007/ BF01537611.

Pettit, G. S., Laird, R. D., Dodge, K. A., Bates, J. E., y Criss, M. M. (2001). Antecedents and behavior-problem outcomes of parental monitoring and psychological control in early adolescence. Child Development, 72(2), 583-598.

Pino, A., Menchón, J. M., Mataix-Cols, D., Pifarré, J., Urretavizcaya, M., Crespo, Jiménez, S., Vallejo, G., y
Vallejo, J. (2004). Perceived parental rearing style in obsessive-compulsive disorder: relation to symptom dimensions. Psychiatry Research, 12, 267-278. doi: 10.1016/j.psychres.2001.12.002.

Quezada, J. (2015). Estilos de crianza en familias nucleares con bijos únicos [Tesis de Maestría]. Cuenca, Ecuador: Universidad de Cuenca. Obtenido de http://dspace. ucuenca.edu.ec/handle/123456789/.

Rodríguez, R. (2010). Juventud, familia y posmodernidad: (des)estructuración familiar en la sociedad contemporánea. Fermentum. Revista Venezolana de Sociología y Antropología, 20(57), 39-55.

Rohner, R. P., \& Britner, P. A. (2002). Worldwide mental health correlates of parental acceptancerejection: Review of cross-cultural and intracultural evidence. Cross-Cultural Research: The Journal of Comparative Social Science, 36(1), 15-47. https://doi. org/10.1177/106939702129146316.

Roy, A. L., Isaia, A., y Li-Grining, C. P. (2019). Making meaning from money: Subjective social status and young children's behavior problems. Journal of Family Psychology, 33(2), 240-245. https://doi.org/10.1037/ fam000048.

Shek, D. (2000). Differences between fathers and mothers in the treatment of, and relationship with, their teenage children: perceptions of Chinese adolescents. Adolescence, 35, 135-147.

Simons, R. L., y Robertson, J. F. (1989). The impact of parenting factors, deviant peers, and coping style upon adolescent drug use. Family Relations, 38, 273-281. doi: $10.2307 / 585052$.

Slicker, E. K., Picklesimer, B. K., Guzak, A., y Fuller, D. (1998). The relationship of parenting style to older adolescent life-skills development. Young, 13, 227-245.

Smetana, J. G. (1995). Parenting style and conceptions of parental authority during adolescence. Child Development, 66, 299-316.

Stattin, H., y Kerr, M. (2000). Parental monitoring: A reinterpretation. Child Development, 71, 1072-1085.

Trenas, A., Osuna, M., Olivares, R., y Cabrera, J. (2013). Relationship between parenting style and aggression in a spanish children sample. Procedia-Social and Behavioral Sciences, 82, 529-536. Obtenido de http://www.sciencedirect.com/science/article/pii/ S1877042813013712. 
Urra, J. (2006). El pequeño dictador. Cuando los padres son las víctimas. Madrid: La Esfera de los Libros.

Van Heel, M., Van Den Noortgate, W., Bijttebier, P., Colpin, H., Goossens, L., Verschueren, K., y Van Leeuwen, K. (2019). Parenting and externalizing problem behavior in adolescence: Combining the strengths of variable-centered and person-centered approaches. Developmental Psychology, 55(3), 653-673. https://doi.org/10.1037/dev0000644.supp.

Vásquez, V. (2015). Estilos de crianza en familias monoparentales con hijos únicos [Tesis de Maestría]. Cuenca, Ecuador: Universidad de Cuenca. Obtenido de http://dspace. ucuenca.edu.ec/handle/123456789/21714.

Vite, A., y Pérez, M. (2014). El papel de los esquemas cognitivos y estilos de parentales en la relación entre prácticas de crianza y problemas de comportamiento infantil. Avances en Psicología Latinoamericana, 32(3), 389-
402. Obtenido de http:/ /www.scielo.org.co/pdf/apl/ v32n3/v32n3a03.pdf.

Wetterborg, D., Enebrink, P., Lönn, K., Forster, M., Risto, E., Dahlström, J., y Ghaderi, A. (2019). A pilot randomized controlled trial of Internet-delivered parent training for parents of teenagers. Journal of Family Psychology, 33(7), 764-774. https://doi.org/10.1037/ fam0000541.sup.

Wittig, S. M. O., y Rodriguez, C. M. (2019). Emerging behavior problems: Bidirectional relations between maternal and paternal parenting styles with infant temperament. Developmental Psychology, 55(6), 11991210. https://doi.org/10.1037/dev0000707.

Woolfolk, A. (2010). Psicología educativa (11a. ed., trad. L. Pineda Ayala). México: Pearson Educación de México. Recuperado de: https:/ / crecerpsi.files.wordpress. com/2014/03/libro-psicologia-educativa.pdf.

Cómo citar este artículo:

Abellán Roselló, L. (2021). Relación entre modelos educativos parentales y problemas de conducta en estudiantes. IE Revista de Investigación Educativa de la REDIECH, 12, e1120. doi: https://doi.org/10.33010/ie_rie_rediech.v12i0.1120. 